\title{
Periodic Electron Structures in Gases: A Fluid Model of the "Window" Phenomenon
}

\author{
P. Nicoletopoulos ${ }^{1}$ and R. E. Robson ${ }^{2, *}$ \\ ${ }^{1}$ Faculté des Sciences, Université Libre de Bruxelles, 1050 Brussels, Belgium \\ ${ }^{2}$ Centre for Antimatter-Matter Studies, Australian National University, Canberra 2600, Australia
}

(Received 22 October 2007; published 25 March 2008)

\begin{abstract}
Periodic electron spatial structures in gases occur within a window of voltages and pressures. Recent accurate solutions of Boltzmann's equation portray this effect, but offer little physical insight into the causes of windowing. Here we show for the first time how such insight can be obtained using the fluid model established by Robson, White, and Petrović [Rev. Mod. Phys. 77, 1303 (2005)], with an appropriate generalization of the heat flux ansatz. Conversely, the success in portraying windowing itself becomes a stringent test of the integrity of this fluid model, which can then be applied to a wider range of problems.
\end{abstract}

Introduction. - The recent resurgence of interest in periodic electron structures in low-current, low-pressure discharges in gases [1-6] has been largely driven by the richness and novelty of the associated physics, in particular, the "window" phenomenon, a sharply defined range of voltages and gas pressures, within which electron properties oscillate, and outside of which properties simply decay monotonically in space, as illustrated in Fig. 5 of Ref. [3]. The existence of the window is not just of theoretical interest, for it is only inside this range that luminous layers [7,8] and periodic density structures [9] can be observed, or the landmark Franck-Hertz experiment [10] can function.

In essence all such macroscopic oscillatory structures are reflections of the electron impact cross sections (both elastic and inelastic), the threshold energies for inelastic collisions, the magnitude of the applied electric field, and the gas pressure. How these then combine to produce the window phenomenon is quite another matter, which needs to be explained, and is the subject of this Letter. In particular, we ask: Why does this window occur, and what factors determine its extent?

The rigorous link between the microscopic and macroscopic pictures, as described above, is provided by Boltzmann's kinetic equation, which one solves for the electron phase space distribution function $f(\boldsymbol{r}, \boldsymbol{v}, t)$ and obtains quantities of physical interest as velocity "moments" $[1-6,11]$. The solution must proceed numerically in phase space $(\boldsymbol{r}, \boldsymbol{v})$ without approximation or decomposition in terms of a density gradient expansion [11].

By their very nature, such rigorous solutions are opaque from a physical point of view, and in any case are accessible to the specialist only. On the other hand one has mean free path analysis, which, while still used in the modern literature [12], is far too crude to offer any hope of dealing with these issues. Thus one is led to ask if there is a theoretical medium, intermediate between the full Boltzmann treatment and simplistic mean free path analysis, which is capable of describing periodic structures. In this paper we show for the first time how a fluid model can be constructed to satisfy this requirement.
Our model is constructed first and foremost with basic atomic and molecular collision physics in mind, but also draws on the ideas of modern low-temperature plasma physics, and otherwise generalizes the theory presented in a recent review article [13] to include inelastic collisions. In this approach, one takes a "short cut", through formulation of approximate balance equations from appropriate velocity moments of Boltzmann's equation. These yield the physical quantities of interest directly, rather than via $f(\boldsymbol{r}, \boldsymbol{v}, t)$, though at the cost of significantly reduced accuracy. As pointed out in Ref. [13], a careful and systematic approach, particularly in regard to the heat flux ansatz, is required to reproduce physical reality — ad hoc models, which abound in plasma physics, have no hope of achieving this end. In this Letter, the benchmarked heat flux ansatz of Ref. [13] is extended to include inelastic collisions, yielding a fluid model which reproduces the essential elements of periodic structures and the associated window phenomenon. We further emphasize that although the specific application here is to periodic electron structures, the ultimate goal is to build a general model, capable of dealing with a wide variety of circumstances.

The fluid model. - In what follows, we will take the idealized steady state Townsend arrangement, i.e., plane parallel geometry, with the electric field $\boldsymbol{E}$ and spatial variations in a direction (say the $z$ axis) normal to the electrodes. The balance equations for particle number, momentum and energy are as in Ref. [13]:

$$
\begin{gathered}
\frac{\partial \Gamma}{\partial z}=0 \\
\frac{2}{3} \frac{\partial(n \varepsilon)}{\partial z}=n q E-n m \nu_{m}(\varepsilon) v \\
\frac{-1}{\nu_{e}}\left(v \frac{\partial \varepsilon}{\partial z}+\frac{2 \varepsilon}{3} \frac{\partial v}{\partial z}+\frac{1}{n} \frac{\partial J}{\partial z}\right)=\varepsilon-\frac{3}{2} k T-\frac{1}{2} M v^{2}+\Omega(\varepsilon)
\end{gathered}
$$

in which the meaning of the symbols is as follows: $q, m$, are the electron charge and mass; $M, T$, are the atomic 
mass and temperature of the gas; $k$ is Boltzmann's constant; $n, v, \varepsilon$, are the electron number density, mean velocity, and mean energy; $\Gamma$ is the electron flux; $J$ is the heat flux; $\Omega=\Sigma_{i} \varepsilon_{i}\left(\vec{\nu}_{i}-\bar{\nu}_{i}\right) / \nu_{e}$ is the energy transferred in inelastic collisions during one elastic energy relaxation time, where $\varepsilon_{i}$ are inelastic thresholds, and $\nu_{e}, \nu_{m}, \vec{\nu}_{i}, \overleftarrow{\nu}_{i}$, are average collision frequencies for energy transfer, momentum transfer, inelastic, and superelastic processes, respectively.

Boundary conditions are such that $v$ and $\varepsilon$ are specified at $z=0$, and that the spatial derivatives of all quantities vanish as $z \rightarrow \infty$.

The collision terms on the right hand side of Eqs. (2) and (3) are calculated according to the lowest order of momentum transfer theory [11], but otherwise the left hand sides are exact. For present purposes we neglect superelastic collisions. The elastic collision frequencies are given by $\nu_{e}=(2 m / M) \nu_{m}, \nu_{m}=N(2 \varepsilon / m)^{1 / 2} \sigma_{m}, N$ being the density of ground state atoms and $\sigma_{m}$ the momentum transfer cross section. The averages $\vec{\nu}_{i}(\varepsilon)$ must be prescribed more carefully in terms of the procedure described in Sec. 7.2 of Ref. [11]. Thus, for one inelastic process of threshold $\varepsilon_{i}$, governed by a constant cross section $\sigma_{i}$, the function $\Omega$ becomes

$$
\Omega(\varepsilon)=\frac{M}{2 m} \varepsilon_{i} \frac{\sigma_{i}}{\sigma_{m}(\varepsilon)} S\left(\frac{3 \varepsilon_{i}}{2 \varepsilon}\right),
$$

where the "smoothing" function $S(\varphi) \equiv(1+\varphi) e^{-\varphi}$ accounts for any sharp jump in $\sigma_{i}$ at threshold [11].

Equations (1)-(3) are closed through the same heat flux ansatz as in Ref. [13]:

$$
J=-\frac{2}{3 m} \frac{\partial}{\partial z}\left[\frac{n \xi(\varepsilon)}{\nu_{m}(\varepsilon)}\right]+\frac{(5-2 p)}{3} \frac{n q E \varepsilon}{m \nu_{m}(\varepsilon)}-\frac{5}{3} \Gamma \varepsilon,
$$

in which $p=d \ln \nu_{m} / d \ln \varepsilon$. For elastic collisions we can set $\xi=\alpha_{0} \varepsilon^{2}$, where $\alpha_{0} \approx 1$, as explained in Ref. [13], but when inelastic collisions are significant, a more general expression must be prescribed. Since $\xi$ has the dimensions of energy squared, the only way [13] to construct such a function is through some combination of both $\Omega$ and $\varepsilon$. Here we make the additional, empirical ansatz,

$$
\xi=\alpha_{0} \varepsilon^{2}\left[1+\frac{\Omega(\varepsilon)}{\varepsilon}\right]^{-r},
$$

where $r$ is an adjustable parameter, an expression which is justified through the benchmarking procedure described below, and which reduces to the established result [13] in the elastic limit $\Omega \rightarrow 0$.

Asymptotic region: The cubic equation. - Again following Ref. [13], we consider the asymptotic region, far downstream from the source, where all quantities are assumed to be only slightly perturbed from their equilibrium expressions, e.g., $n(z)=n_{\infty}+n_{1} e^{K z}$ and similarly for $v(z), \varepsilon(z)$ and $J(z)$. Attention is focused on the decay constant $K$, expressed in terms of a dimensionless quantity $\kappa$ by $K=$
$(3 / 2)\left(q E / \varepsilon_{\infty}\right) \kappa$. Eqs. (1)-(3) and (5) are then linearized in small quantities leading to four homogeneous equations in the four unknowns, $n_{1}, v_{1}, \varepsilon_{1}, J_{1}$. This system has a nontrivial solution if and only if $\kappa$ satisfies the cubic equation

$$
\begin{aligned}
\frac{3}{2} \alpha(\bar{p}-p-1) \kappa^{3}-\left(\frac{3}{2} \alpha \bar{p}-\frac{5}{2} p+p^{2}\right) \kappa^{2} \\
-\left(\gamma+2 p-\frac{7}{2}\right) \kappa+(\gamma+2 p)=0,
\end{aligned}
$$

in which

$$
\begin{gathered}
\bar{p}=\frac{\varepsilon_{\infty} \xi^{\prime}\left(\varepsilon_{\infty}\right)}{\xi\left(\varepsilon_{\infty}\right)}=2+r(1-\gamma), \\
\alpha=\frac{\xi\left(\varepsilon_{\infty}\right)}{\varepsilon_{\infty}^{2}}=\alpha_{0}\left[1+\frac{\Omega\left(\varepsilon_{\infty}\right)}{\varepsilon_{\infty}}\right]^{-r}, \\
\gamma=\frac{\left(1+\Omega^{\prime}\right) \varepsilon_{\infty}}{\varepsilon_{\infty}+\Omega-(3 / 2) k T} .
\end{gathered}
$$

Physically meaningful solutions of Eq. (7) are prescribed by $\operatorname{Re}(\kappa) \leq 0$. For purely elastic collisions, $\Omega=0, \gamma=1$, and the solutions of (7) are always real; i.e., there are no oscillations. When inelastic collisions are important, $\gamma$ can significantly exceed unity, and the solutions of (7) can be complex, leading to oscillatory behavior and window structures, as we now explain.

Benchmark model. - The Boltzmann equation has been solved numerically [3] for constant cross sections $\sigma_{m}=$ $6 \AA^{2}$, and $\sigma_{i}=0.1 \AA^{2}$ above threshold, $\varepsilon_{i}=2 \mathrm{eV}$, the gas having $M=4$ a.m.u., and $T=0$. We now test our fluid theory by computing solutions of the cubic equation for this model $(p=0.5)$. The parameter $\alpha_{0}$ is fixed by requiring that for elastic scattering, i.e., $\gamma=1, \bar{p}=2$, the solution coincides with the Boltzmann equation result (see Ref. [13], Sec. B.2.c). The value of $r$ is determined by trial and error for good agreement in the inelastic region. Figure 1 shows plots of $-\operatorname{Re}\left(K^{*}\right)$ and $\operatorname{Im}\left(K^{*}\right)$ as functions of $E / N$, where $K^{*}$ is the dimensionless quantity $K \lambda, \lambda$ being a length scale defined in Fig. 1. It is clear that there is a window of reduced fields, roughly $0.5 \mathrm{Td}<E / N<$ $10 \mathrm{Td}$, within which the real part of $K^{*}$ is small, and the imaginary part rises monotonically, implying that periodic structures persist to large distances downstream from the source, as illustrated in Fig. 2 for mean energy. Figure 1 is in reasonable quantitative agreement with the rigorously derived result [see [3], Fig. 4(a)]. This is the first time that such behavior has been produced using a fluid model. Using the physically-based heat flux ansatz (5) is critical to the exercise. Certain popular, but deeply flawed ad hoc expressions for $J$ [13], have no chance of reproducing these effects. 


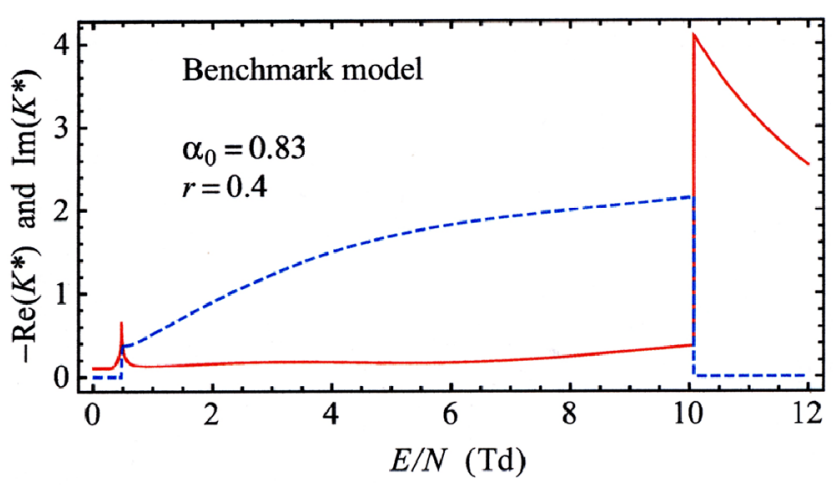

FIG. 1 (color online). Real (solid line), and imaginary (dashed line), parts of the dimensionless wave number $K^{*}=K \lambda$, as functions of $E / N$, as calculated for the benchmark model described in the text. In this and the following figures, length is scaled according to $\lambda=\left(2^{1 / 2} N \sigma_{0}\right)^{-1}$ with $\sigma_{0}=1 \AA^{2}$, and $1 \mathrm{Td}=1$ townsend $=10^{-21} \mathrm{~V} \mathrm{~m}^{2}$.

A physical understanding of the window phenomenon, and the key role played by $\gamma$, then follows: (i) If $E / N$ is so small that inelastic collisions are negligible, then $\gamma \approx 1$, and the cubic equation has only real solutions. This is a mathematical reflection of the fact that elastic collisions provide no physical mechanism to produce oscillations. (ii) As the value of $E / N$ increases, inelastic collisions become significant, and physically we would expect oscillations to occur at some stage. Consistently with this, we find that in all cases $\gamma(E / N)$ increases above unity, as in Fig. 3, and-if the quantity

$$
\rho=(M / m)\left(\sigma_{i} / \sigma_{m}\right)
$$

is sufficiently large $-\gamma$ eventually exceeds some critical value $\gamma_{c}$, at which point complex solutions of Eq. (7) become possible, leading to oscillations. (iii) As $E / N$ is further increased, the relative importance of inelastic processes declines: the magnitude of energy loss in elastic collisions keeps on increasing but the energy lost in inelastic processes remains fixed. We thus expect that oscilla-

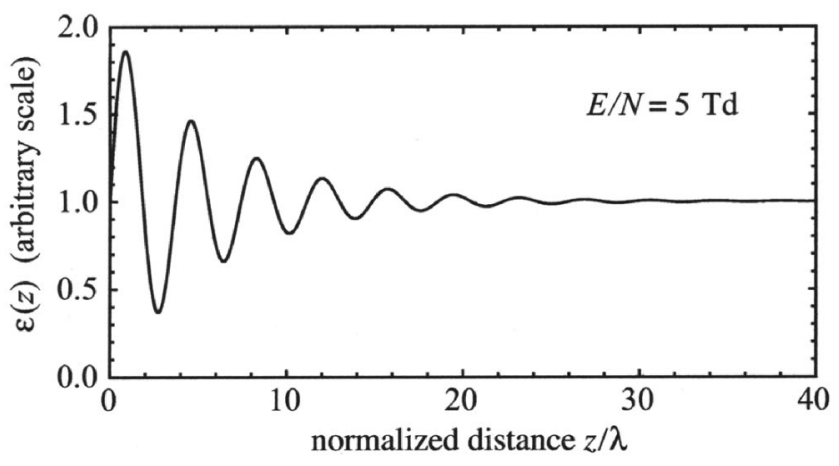

FIG. 2. Mean energy at $E / N=5 \mathrm{Td}$ as a function of normalized distance downstream from the source. The scale length $\lambda$ is defined in Fig. 1 tions would eventually become damped as $E / N$ increases. Again this is reflected in the behavior of $\gamma(E / N)$ which attains a maximum value $\gamma_{\max }$, and then falls below $\gamma_{c}$ as $E / N$ increases. Oscillations thus occur in a window of $E / N$ corresponding to $\gamma_{c}<\gamma<\gamma_{\max }$, as marked in Fig. 3. (iv) While the value of $\rho$, and hence $\gamma_{\max }$, is determined in advance by specifying the atomic parameters and cross sections, the corresponding value of $\gamma_{c}$ emerges only after solution of Eq. (7). In actual fact, $\gamma_{\max }$ increases and $\gamma_{c}$ decreases with increasing $\rho$, producing an expanded window. Conversely, reducing $\rho$ lowers $\gamma_{\max }$ and raises $\gamma_{c}$ resulting in a shrinking window. We emphasize that $\gamma_{\max }$ is determined entirely by $\rho$-if this is so small that $\gamma_{\max }<\gamma_{c}$, then, regardless of the magnitude of the threshold energy $\varepsilon_{i}$, oscillations cannot occur at any $E / N$. (v) Since elastic collisions play an important role in moderating energies and randomizing the velocities of electrons, then neither $m / M$ nor $\sigma_{m} / \sigma_{i}$ can be too small, and Eq. (11) implies an upper bound on $\rho$. (The limits $M / m \rightarrow \infty$, or $\sigma_{m} / \sigma_{i} \rightarrow 0$, i.e., $\rho \rightarrow \infty$, implicit in typical textbook explanations of the Frank-Hertz experiment, are clearly problematic [3].) Both upper and lower bounds are independent of $\varepsilon_{i}$, with some minor influence through $\alpha_{0}$ and $r$. For the present case $\left(\alpha_{0}=0.83, r=0.4\right)$, the bounds are $11.5<\rho<300$. (vi) The breadth of the peak in $\gamma(E / N)$ - and hence the width of the window - is proportional to the threshold energy $\varepsilon_{i}$, since the larger its value, the longer the influence of the inelastic collisions lasts [see point (iii) above]. Conversely, reducing $\varepsilon_{i}$ tends to suppress the width of the window, which is negligible if $\varepsilon_{i}$ is small enough. This is why in molecular gases, where typically $\varepsilon_{i} \ll 1 \mathrm{eV}$ for rotational excitations, oscillations are not often observed [9].

For the step function benchmark model, $\rho \approx 122$, $\gamma_{\max } \approx 4.5$ and, $\gamma_{c} \approx 1.6$, and as Fig. 3 shows, there is indeed a window of oscillations in the range

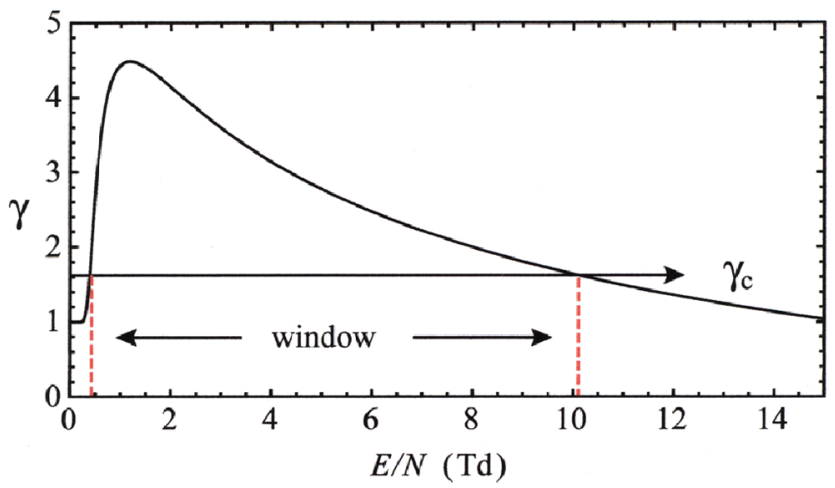

FIG. 3 (color online). The parameter $\gamma$ for the step function model, showing how the window of $E / N$ is defined when the maximum $\left(\gamma_{\max }\right.$ in the text) of the curve $\gamma(E / N)$ is above a certain critical value $\gamma_{c}$ (horizontal line). The value of $\gamma_{c}$ emerges after solution of the cubic. Note that for purely elastic collisions, $\gamma=1$ for all $E / N$ and no oscillations are possible. 

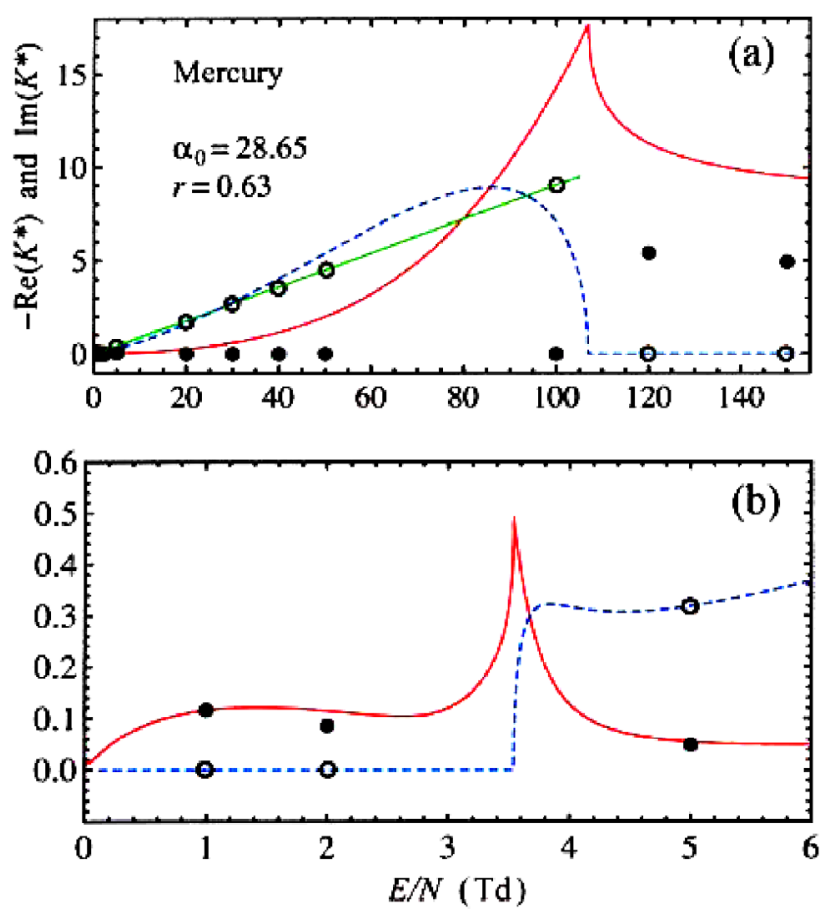

FIG. 4 (color online). (a) Real and imaginary parts of the dimensionless wave number $K^{*}=K \lambda$, as functions of $E / N$ for mercury, as calculated by fluid model and from Boltzmann's equation [3,15]. $-\operatorname{Re}\left(K^{*}\right)$ : solid curve (fluid) and disks (Boltzmann). $\operatorname{Im}\left(K^{*}\right)$ : dashed line (fluid) and circles (Boltzmann). Straight line corresponds to the "ideal" period of $4.9 \mathrm{eV}$; for $\operatorname{Im}\left(K^{*}\right)$ above this line the period is shorter and vice versa. (b) Detailed view at low $E / N$.

$0.5 \mathrm{Td}<E / N<10 \mathrm{Td}$. This range is consistent with the Boltzmann equation results [3], and we can therefore have confidence in both the soundness of the above physical arguments and in the integrity of the fluid model.

Periodic structures in real gases. - In order to deal with more realistic examples, we computed the physical solutions of the cubic equation using the true energy-dependent momentum transfer cross section $\sigma_{m}(\varepsilon)$ and a reasonable constant "bulk" inelastic cross section $\sigma_{i}$ above the lowest important threshold $\varepsilon_{i}$. Note that now the parameter $p$ is energy dependent.

Figure 4 shows fluid and Boltzmann equation results for the real and imaginary parts of $K^{*}$ for mercury gas $(M=$ 200 a.m.u., $T=0$ ) calculated with $\sigma_{i}=2 \AA^{2}, \varepsilon_{i}=$ $4.9 \mathrm{eV}$, using the analytic expression for $\sigma_{m}(\varepsilon)$ derived in Ref. [14] in terms of generalized Fano profiles. Some Boltzmann equation results for $\mathrm{Hg}$ were first reported in Ref. [3], but the detailed data shown here are due to Li [15]. The parameter $\alpha_{0}$ is determined by matching the purely elastic fluid solution at $E / N=1 \mathrm{Td}$, to the rigorous data [see Fig. 4(b)]. The overall agreement of fluid and kinetic theory results is fairly good, with a notable exception in regard to the real part in the upper extremity of the window. An abnormally large inaccuracy specific to the real part is also observed in the benchmark model; as of this writing the origin of this discrepancy is not understood.

Conclusion. - We have formulated for the first time a fluid model capable of describing periodic electron structures in a constant electric field, and, in particular, reproducing the window phenomenon. The extent of the window is controlled by the function $\gamma$ of Eq. (10), which in turn is sensitive to the mass and cross section ratios as well as to the magnitude of the threshold energy. The calculations reported here have been for the asymptotic regime downstream from the source, but the fluid model is capable of covering the entire region, and can readily be extended to other geometries. The key factor in this successful outcome is the established heat flux ansatz, Eq. (5), and its subsidiary (6). The present results can be seen as providing not only an explanation of the window effect per se, but also as furnishing a fluid model of some generality, whose integrity has been stringently tested in a demanding problem. This Letter thus demonstrates a positive synergism linking fundamental atomic collision physics on the one hand, and plasma fluid modeling on the other.

The support of the Australian Academy of Science is gratefully acknowledged. We thank Dr. Bo Li for providing the Boltzmann equation data in Figure 4 and Dr. Ronald White for helpful discussions.

*robert.robson@anu.edu.au

[1] G. Petrov and R. Winkler, J. Phys. D 30, 53 (1997).

[2] R. Winkler, V. A. Maiorov, and F. Sigeneger, J. Appl. Phys. 87, 2708 (2000).

[3] R. E. Robson, B. Li, and R. D. White, J. Phys. B 33, 507 (2000).

[4] B. Li, R. D. White, and R. E. Robson, J. Phys. D 35, 2914 (2002).

[5] F. Sigeneger, R. Winkler, and R.E. Robson, Contrib. Plasma Phys. 43, 178 (2003).

[6] B. Li, R.E. Robson, and R. D. White, Phys. Rev. E 74, 026405 (2006).

[7] G. Holst and E. Oosterhuis, Physica (Amsterdam) 1, 78 (1921).

[8] M. J. Druyvesteyn, Z. Phys. 73, 33 (1932).

[9] J. Fletcher, J. Phys. D 18, 221 (1985).

[10] J. Franck and G. Hertz, Verh. Deutsche Phys. Ges. 16, 457 (1914).

[11] R. E. Robson, Introductory Transport Theory for Charged Particles in Gases (World Scientific, Singapore, 2006).

[12] G. Rapior, K. Sengstock, and V. Baev, Am. J. Phys. 74, 423 (2006).

[13] R. E. Robson, R. D. White, and Z. Lj. Petrovic, Rev. Mod. Phys. 77, 1303 (2005).

[14] P. Nicoletopoulos, http://arxiv.org/abs/physics/0307081.

[15] B. Li (private communication). 